\section{Brain, Behavior and Evolution}

Abellán, A. 112

Alpár, A. 82

Bácskai, T. 104

Balázsa, T. 140

Bandín, S. 93

Birinyi, A. 104

Csillag, A. 81

de Carlos, J.A. 126

Desfilis, E. 112

Fritzsch, B. 150, 162

Gáti, G. 82

Glover, J.C. 162
González, A. 93

Gray, D. 140

Harkany, T. 82

Hevner, R.F. 126

Hökfelt, T. 82

Kaas, J.H. 126

Kecskes, S. 104

Köppl, C. 150

Lein, E. 126

Lendvai, D. 82

López, J.M. 93

Matesz, K. 104

Medina, L. 112
Molnár, Z. 126

Montagnese, C.M. 140

Moreno, N. 93

Morona, R. 93

Němec, P. 126

Rácz, É. 104

Sienknecht, U.J. 150

Straka, H. 162

Székely, T. 140

ten Donkelaar, H.J. 81

Vicario, A. 112

Zachar, G. 140

\title{
Subject Index Vol. 83, No. 2, 2014
}

Basal ganglia 93

Basilar papilla 150

Bed nucleus of the stria terminalis 140

Brain evolution 93

Brainstem 104

Comparative neuroanatomy 82, 93

- transcriptomics 126

Cortex 82

Cortical arealization 126

- neurogenesis 126

Developmental regulatory genes 112

Ear evolution 162

Efferent development 150

Enkephalin 112

Evolution 82

Eye muscle evolution 162

Forebrain evolution 112
Globus pallidus 112

Hair cell orientation 150

Hypoglossal nerve 104

Immunohistochemistry 93

Inner ear efferent evolution 150

Intermediate progenitors 126

Islet1 112

Lateral olivocochlear efferents 150

- septal areas 140

Medial olivocochlear efferents 150

- preoptic nucleus 140

Nkx2.1 112

Octavolateral efferents 150
Pallidostriatal projections 112

Pallidum 93, 112

Pallium 82

Planar cell polarity 150

Prey-catching behavior 104

Sensorimotor integration 104

Sensory epithelia 150

Songbirds 140

Striatum 93, 112

Subterranean vision 126

Tangential neuronal migration 126

Vasoactive intestinal peptide 140

Vasotocin 140

Vestibular efferents 150

- nucleus 162

- projection 162

Vestibulo-ocular reflex 162

Wnt signaling 150 